\title{
The Neutrino Decay of the Free Neutron and the Neutrino Structure According to the Planck Vacuum Theory
}

\author{
William C. Daywitt
}

\begin{abstract}
The Planck vacuum (PV) theory derives equations for the neutrino and antineutrino, and relates them to the unstable free neutron and antineutron. Remarkably, these neutrons and neutrinos share the same wave function solutions that describe the proton and electron and their antiparticle cores.

The neutrino and antineutrino are chargeless and massless; so their propagation through matter goes unnoticed, making these neutrinos invisible. The equations to follow that describe these pseudo-particles are the theoretical embodiment of the circa 1930 Pauli neutrino hypothesis.

Finally, depending on one's perspective, the neutrons can be viewed as decaying meta-particles or as stable nuclear particles.
\end{abstract}

Index Terms-Neutron Decay, Neutrino Structure, Nuclear Neutrons, Planck Vacuum State.

\section{INTRODUCTION}

$\mathbf{T}$ HIS paper is a continuation of an earlier neutron paper [1], where here the neutrino is introduced into the PV theory. That theory recognizes a 7-dimensional (7-D) spacetime that consists of two 4-dimensional (4-D) spacetimes, a 4-D observed spacetime and a 4-D unobserved space time. The particles and antiparticles of equations (6)-(9) reside in the observed and unobserved 4-D spacetimes respectively.

The theoretical foundation [2] [3] [4] [5] of the PV theory rests upon the unification of the Einstein, Newton, and Coulomb super forces:

$$
\frac{c^{4}}{G}\left(=\frac{m_{*} c^{2}}{r_{*}}\right)=\frac{m_{*}^{2} G}{r_{*}^{2}}=\frac{e_{*}^{2}}{r_{*}^{2}} \rightarrow r_{*} m_{*} c=\frac{e_{*}^{2}}{c} \quad(=\hbar)
$$

where the ratio $c^{4} / G$ is the curvature superforce that appears in the Einstein field equations. $G$ is Newton's gravitational constant, $m_{*}$ and $r_{*}$ are the Planck mass and length respectively [6, p.1234], and $e_{*}$ is the massless bare (or coupling) charge. The fine structure constant is given by the ratio $\alpha \equiv e^{2} / e_{*}^{2}$, where $e$ is the observed electronic charge magnitude. The ratio $e_{*}^{2} / c$ to the right of the arrow is the spin coefficient for the Planck particle (PP), the proton, and the electron cores respectively, where $\hbar$ is the reduced Planck constant.

The two particle/PV coupling forces

$$
F_{e}(r)=\frac{e_{*}^{2}}{r^{2}}-\frac{m_{e} c^{2}}{r} \quad \text { and } \quad F_{p}(r)=\frac{e_{*}^{2}}{r^{2}}-\frac{m_{p} c^{2}}{r}
$$

DOI: http://dx.doi.org/10.24018/ejers.2021.6.5.2524

Published on July 27, 2021.

William C. Daywitt is retired from the National Institute of Standards and Technology,Boulder, CO 80305 USA. (e-mail: wcdaywitt@me.com). the electron core $\left(-e_{*}, m_{e}\right)$ and proton core $\left(+e_{*}, m_{p}\right)$ exert on the invisible PV state; along with their coupling constants

$$
F_{e}\left(r_{e}\right)=0 \text { and } F_{p}\left(r_{p}\right)=0
$$

and the resulting Compton radii

$$
r_{e}=\frac{e_{*}^{2}}{m_{e} c^{2}}=\frac{e_{*}^{2} / c}{m_{e} c} \quad \text { and } \quad r_{p}=\frac{e_{*}^{2}}{m_{p} c^{2}}=\frac{e_{*}^{2} / c}{m_{p} c}
$$

lead to the important string of Compton relations

$$
r_{e} m_{e} c=r_{p} m_{p} c=\frac{e_{*}^{2}}{c}=r_{*} m_{*} c \quad(=\hbar)
$$

for the electron and proton cores. The PP Compton radius is $r_{*}=e_{*}^{2} / m_{*} c^{2}$, derived by equating the Einstein and Coulomb super forces from (1). To reiterate, the equations in (2) represent the forces the free electron or proton cores exert on the invisible PV space, a space that is itself pervaded by a degenerate collection of PP cores $\left( \pm e_{*}, m_{*}\right)$. The positron and antiproton cores are $\left(+e_{*}, m_{e}\right)$ and $\left(-e_{*}, m_{p}\right)$ respectively.

The $e_{*}^{2}$ for the left sides of (6)-(9) is the squared coupling charge, where one of the $e_{*} \mathrm{~s}$ belongs to the free particle core and the other charge belongs to any one of the PP cores making up the degenerate PV state.

After the Introduction in Section I, Section II reviews the Dirac core equations for the electron and proton. Section III reintroduces and updates the neutron particle equations from [1] by introducing (in Section IV) the neutrino into the PV calculations. Section V concerns the nuclear neutron and the stability of the atomic nucleus.

\section{DiRAC CORES}

The covariant Dirac equation leads to the following four $2 \mathrm{x} 1$ spinor equations [5], (where $x^{0}=c t$ and the sum is over $j=1,2,3$ )

$$
\begin{gathered}
i \frac{e_{*}^{2}}{c}\left(u^{\prime}, v^{\prime}\right)=m_{e} c u^{\prime} \\
-i \frac{e_{*}^{2}}{c}\left(v^{\prime}, u^{\prime}\right)=m_{e} c v^{\prime}
\end{gathered}
$$

and

$$
\begin{gathered}
i \frac{e_{*}^{2}}{c}\left(u^{\prime \prime}, v^{\prime \prime}\right)=m_{p} c u^{\prime \prime} \\
-i \frac{e_{*}^{2}}{c}\left(v^{\prime \prime}, u^{\prime \prime}\right)=m_{p} c v^{\prime \prime}
\end{gathered}
$$

which, from top to bottom, describe the electron, positron, proton, and antiproton cores respectively. The $u$ s and $v$ s are the 
$2 \times 1$ spinor wave function solutions to the equations. Equations (6)-(9) are spin angular-momentum equations, including the spin coefficient $e_{*}^{2} / c$ on the left and the various spin momenta $m_{e} c$ etc. on the right. The parenthetical gradient operators on the left are defined in Appendix A.

The ratio $e_{*}^{2} / c$ is the spin coefficient, where

$$
\vec{S}=\frac{e_{*}^{2}}{c} \vec{\sigma} \quad \longrightarrow \quad \frac{e_{*}^{2}}{c} \sigma_{j} \frac{\partial}{\partial x^{j}}
$$

is the relativistic spin of the electron or proton cores. The $2 \times 2$ Pauli spin vector is $\vec{\sigma}$. The second expression is the scalar-product sum of $\vec{S}$ with the gradient operator $\partial / \partial x^{j}$; that is, the PV gradient $\partial / \partial x^{j}$ in the $j$ th direction weighted by the relativistic spin in that direction. As seen in (5): the spin magnitudes of the PP, the proton, and the electron cores are identical (the spin of their antiparticles is the negative of these).

Superposition, i.e. adding the separate components $\left[e_{*}^{2} / c, u, v, m_{e}, m_{p}\right]$ from the electron and proton cores (6)-(9), leads to:

$$
\overbrace{i\left(\frac{e_{*}^{2}}{c}-\frac{e_{*}^{2}}{c}\right)}^{0}\left(u^{\prime}+v^{\prime}, v^{\prime}+u^{\prime}\right)=m_{e} c\left(u^{\prime}+v^{\prime}\right)=m_{e} c \widehat{0}
$$

for the electron-positron, and

$$
\overbrace{i\left(\frac{e_{*}^{2}}{c}-\frac{e_{*}^{2}}{c}\right)}^{0}\left(u^{\prime \prime}+v^{\prime \prime}, v^{\prime \prime}+u^{\prime \prime}\right)=m_{p} c\left(u^{\prime \prime}+v^{\prime \prime}\right)=m_{p} c \widehat{0}
$$

for the proton-antiproton, where

$$
\left(u^{\prime}+v^{\prime}\right)=\widehat{0} \quad \text { and } \quad\left(u^{\prime \prime}+v^{\prime \prime}\right)=\widehat{0}
$$

are the $2 \times 1$ null spinor solutions to (11) and (12). Equations (11) and (12) constitute the electron and proton annihilation equations in the PV theory-reflecting the experimental fact that the core and anticore form a particle-antiparticle pair. It is noted that the spinor gradients on the left sides of (11) and (12) do not necessarily vanish.

\section{Neutron AND Antineutron EQuATIONS}

The superposition of the proton and the positron

$$
\overbrace{\left(\frac{e_{*}^{2}}{c}-\frac{e_{*}^{2}}{c}\right)}^{0}\left[i\left(u^{\prime \prime}+v^{\prime}, v^{\prime \prime}+u^{\prime}\right)\right]=m_{p} c u^{\prime \prime}+m_{e} c v^{\prime}
$$

leads to the free neutron. The corresponding superposition of the antiproton and the electron

$$
\overbrace{\left(\frac{e_{*}^{2}}{c}-\frac{e_{*}^{2}}{c}\right)}^{0}\left[i\left(v^{\prime \prime}+u^{\prime}, u^{\prime \prime}+v^{\prime}\right)\right]=m_{p} c v^{\prime \prime}+m_{e} c u^{\prime}
$$

leads to the free antineutron. These two equations must decay because the differing spin momenta $\left(m_{p} c\right.$ and $\left.m_{e} c\right)$ on their right hand sides cannot lead to a vanishing $2 x 1$ spinor, as in equations (13) for the electron and proton cores.

\section{INTRODUCING THE NEUTRINOS}

From (14) and (15), the neutrino gradient equations that define the neutrinos are

$$
\begin{array}{r}
\text { (neutrino) } \equiv i\left(u^{\prime \prime}+v^{\prime}, v^{\prime \prime}+u^{\prime}\right) \\
=i\left[\frac{\partial\left(u^{\prime \prime}+v^{\prime}\right)}{\partial x^{0}}+\sigma_{j} \frac{\partial\left(v^{\prime \prime}+u^{\prime}\right)}{\partial x^{j}}\right]
\end{array}
$$

and

$$
\begin{aligned}
& \text { (antineutrino) } \equiv i\left(v^{\prime \prime}+u^{\prime}, u^{\prime \prime}+v^{\prime}\right) \\
& =i\left[\frac{\partial\left(v^{\prime \prime}+u^{\prime}\right)}{\partial x^{0}}+\sigma_{j} \frac{\partial\left(u^{\prime \prime}+v^{\prime}\right)}{\partial x^{j}}\right]
\end{aligned}
$$

where the neutrino and antineutrino are chargeless and massless; so their propagation through matter goes unnoticed, making the neutrinos invisible.

Equations (14)-(17) yield

$$
\overbrace{\left(\frac{e_{*}^{2}}{c}-\frac{e_{*}^{2}}{c}\right)}^{0}(\text { neutrino })=m_{p} c u^{\prime \prime}+m_{e} c v^{\prime}
$$

and

$$
\overbrace{\left(\frac{e_{*}^{2}}{c}-\frac{e_{*}^{2}}{c}\right)}^{0}(\text { antineutrino })=m_{p} c v^{\prime \prime}+m_{e} c u^{\prime}
$$

for the two 4-D free neutron and antineutron decay equations, which will decay in less than fifteen minutes.

Adding equations (18) and (19) before their decay time expires, and using (13), leads to

$$
\begin{gathered}
\overbrace{\left(\frac{e_{*}^{2}}{c}-\frac{e_{*}^{2}}{c}\right)}^{0}[(\text { neutrino })+(\text { antineutrino })] \\
=m_{p} c u^{\prime \prime}+m_{e} c v^{\prime}+m_{p} c v^{\prime \prime}+m_{e} c u^{\prime} \\
=m_{p} c\left(u^{\prime \prime}+v^{\prime \prime}\right)+m_{e} c\left(v^{\prime}+u^{\prime}\right)=m_{p} c \widehat{0}+m_{e} c \widehat{0}
\end{gathered}
$$

for the neutron-antineutron annihilation equation.

\section{Comments And Conclusions}

The neutron equation (18) by itself

$$
\overbrace{\left(\frac{e_{*}^{2}}{c}-\frac{e_{*}^{2}}{c}\right)}^{0}(\text { neutrino })=m_{p} c u^{\prime \prime}+m_{e} c v^{\prime}
$$

displays the well-known free neutron-decay pattern seen in the observed 4-D spacetime: the neutron $\beta$-decay leading to the proton and electron scatter velocities for $m_{p}$ and $m_{e}$ that are non-colinear, implying that there must be a third unobserved pseudo-particle (the neutrino on the left) involved in the decay-as noted by Wolfgang Pauli [7, p.531] around circa 1930. 
If the process described by equation (20) is taking place within the confined space of the atomic nucleus (rather than free space), the decay times in (18) and (19) shrink to zero and (20) becomes the annihilation equation for the nuclear neutron and its antineutron. If the stable neutron is then ejected from the nucleus back into free space, it reverts to the meta-state described by the 4-D equation (18) and decays.

Two important conclusions to be drawn from the previous two paragraphs, and from comparing (20) to the electron and proton equations (11) and (12), are that: viewed from a 4-D perspective, the neutrons are meta-particles that decay in less than fifteen minutes; and viewed from a 7-D perspective, the neutrons are stable particles that can now take their place next to the proton particles, and function to stabilize the atomic nucleus.

\section{APPENDIX A \\ GRADIENT OPERATOR}

The gradient operator (summing over $j=1,2,3$ )

$$
(U, V) \equiv\left(\frac{\partial U}{\partial x^{0}}+\sigma_{j} \frac{\partial V}{\partial x^{j}}\right)
$$

is defined for equations of the form of (6)-(9).

\section{REFERENCES}

[1] W.C. Daywitt, The Neutron Meta-Particles and their Decay as Viewed in the Planck Vacuum Theory. European Journal of Engineering Research and Science, VOL. 5, NO. 8, AUGUST 2020. (See also www.planckvacuumDOTcom.)

[2] P. Davies, Superforce: the Search for a Grand Unified Theory of Nature. Simon and Schuster, Inc., New York, 1984.

[3] W.C. Daywitt, "A Model for Davies' Universal Superforce", Galilean Electrodynamics, Sept./Oct., 83, 2006.

[4] W.C. Daywitt, "The Trouble with the Equations of Modern Fundamental Physics", American Journal of Modern Physics. Special Issue: "Physics without Higgs and without Supersymmetry", v. 5, no. 1-1, 22, 2016.

[5] W.C. Daywitt, Comparing the Planck-Vacuum and the Urantia-Book Depictions of the Seven-Dimensional Spacetime, European Journal of Engineering Research and Science, Vol.5, No.12, December, 2020.

[6] B.W. Carroll, D.A. Ostlie, An Introduction to Modern Astrophysics, (Addison-Wesley, San Francisco, Boston, New York, Cape Town, Hong Kong, London, Madrid, Mexico City, Montreal, Munich, Paris, Singapore, Sidney, Tokyo, Toronto, 2007).

[7] R.B. Leighton, Principles of Modern Physics, McGraw-Hill Book Co., New York, Toronto, London, 1959. 